\title{
Whole-genome sequencing reveals high genetic diversity of Streptococcus uberis isolated from cows with mastitis
}

\author{
Nathália Cristina Cirone Silva ${ }^{1,2^{*}}$, Yongqiang Yang ${ }^{3,4}$, Marjory Xavier Rodrigues ${ }^{1}$, Tiago Tomazi ${ }^{1}$ and \\ Rodrigo Carvalho Bicalho ${ }^{1}$
}

\begin{abstract}
Background: Bovine mastitis is an important cause of economic loss in dairy farms. Streptococcus uberis is among the most frequently isolated bacterial species isolated from cows with mastitis. The aim of this study was to perform an indepth genetic assessment of $S$. uberis strains isolated from bovine clinical mastitis (CM) and to perform a phylogenetic analysis to represent the evolutionary relationship among $S$. uberis sequences.

Results: A total of 159 isolates was genetically characterized using whole genome sequencing. According to the virulence determinants, all strains harbored the hasC, leuS, perR, purH, and purN virulence genes. Thirty-four resistance genes were identified in at least one strain. In terms of acquired genes, we observed that 152 (95.6\%) strains had a resistance gene to lincosamine (InuD), $48(30.2 \%)$ to tetracycline (tetM), $4(2.51 \%)$ to tobramicine (ant6), and 1 to lincosamide (Isa(E)). MLST detected the Sequence Type (ST)797 $(n=23)$, while $85.5 \%$ of the strains did not match to known STs.

Conclusions: Then, eleven distinct ST were identified after we submitted the new alleles to assign new STs. The other prevalent STs observed were ST1215 $(n=58)$, ST1219 $(n=35)$, and ST1213 $(n=15)$. And it was not possible to identify the MLST of four strains. Phylogenetic lineages indicated a high genomic diversity of S. uberis in our collection, confirming that most strains isolated from bovine mastitis have different reservoirs, typical of environmental pathogens.
\end{abstract}

Keywords: New generation sequencing, Virulence genes, Phylogenetic tree

\section{Background}

Bovine mastitis is one of the major concerns for the dairy industry being associated with direct and indirect economic losses. Direct losses include the increased costs with veterinary services, milk discard, mortality and culling of animals, and reduction of milk quality. On the other hand, indirect losses are considered the most substantial one, which include the reduction of milk

\footnotetext{
*Correspondence: ncirone@unicamp.br

${ }^{2}$ Department of Food Science and Nutrition, Faculty of Food Engineering

(FEA), University of Campinas (UNICAMP), Rua Monteiro Lobato, 80, SP

13083-862 Campinas, Brazil

Full list of author information is available at the end of the article
}

production, changing in milk composition, pre-term drying-off, impairment of reproductive performance, animal welfare aspects, and other associated health issues $[1,2]$.

Many microbial species were described as the cause of bovine mastitis and among them, Streptococcus spp. is among the most isolated genera in dairy herds, being associated with both clinical and subclinical forms of the disease [3, 4]. Within the Streptococcus genus, Streptococcus uberis is the most prevalent species envolving with bovine mastitis [5]. S. uberis is a Gram-positive pathogen inducing both clinical and subclinical mastitis, causing reduction of milk production, changes in milk composition and increase of somatic cell count (SCC) in dairy 
cows [6-8]. The high polymorphism of strains isolated from bovine mastitis indicates that the environment (e.g. bedding used in housing facilities and pastures) is the main reservoir of S. uberis [9]. However, recent studies have shown evidences that certain strains might be transmitted from cow to cow during milking $[10,11]$. S. uberis was also associated with persistent intramammary infections, which could be related to its ability to internalize in the mammary gland [12], along with its increased resistance to antimicrobials [13, 14]. Despite several studies evaluating $S$. uberis have been published in recent years, the role of this species in the epidemiology of mastitis is not completely understood. With the advent of powerful molecular methods, such as whole genome sequencing, it is now possible to detect genetic antimicrobial resistance determinants and virulence factor genes [15]. The advance of knowledge about the genetic features associated with $S$. uberis causing mastitis associated with clinical outcomes such as cure after antimicrobial treatment, death/culling due mastitis, mammary quarter loss and disease reoccurrence, can contribute to the developing of efficient strategies for prevention and control of this pathogen in dairy herds.

The aim of this study was to perform an in-depth genetic assessment of $S$. uberis isolated from bovine clinical mastitis (CM) and to perform a phylogenetic analysis to represent the evolutionary relationship between $S$. uberis sequences.

\section{Results and discussion Descriptive data}

A total of 159 S. uberis strains were selected from 151 cows. Isolates identified from the same cows were isolated from clinical mastitis occurred in different mammary quarters. Cows from which the strains were isolated had an average number of lactation of $2.6(\mathrm{SD}=1.4)$ and DIM of $119.7(\mathrm{SD}=89.8)$. In total, $83 \%$ of the isolates $(n=132)$ were recovered from mild cases (i.e., only changes in the milk appearance) of clinical mastitis, while $17 \%$ where either moderate (i.e., changes in milk appearance associated with inflammatory symptoms in the udder) or severe (i.e., changes in the milk and udder associated with systemic inflammatory symptoms).

According to cow-level records, the following outcomes were recorded from cows after $\mathrm{CM}$ caused by the $S$. uberis isolates selected herein: mortality after CM (19.5\%), bacteriological cure $(44.65 \%)$, mammary quarter loss $(10.7 \%)$, clinical cure $(87.4 \%)$ and reoccurrence of clinical mastitis (23.9\%).

In Figs. 1 and 2, we demonstrate the relative risk of each encoding gene and the probability of mortality and bacteriological cure after 14 days for cows, respectively. We present the risk, significance, prevalence of positive bacteria and cows infected by them which dye and which alive, and total prevalence of genes in cows.

\section{Virulence factors}

All strains presented five virulence genes: has C, leuS, per $\mathrm{R}, p u r \mathrm{H}$, and $p u r \mathrm{~N}$. They were reported to encode hyaluronic acid capsule (has C), Leucyl-tRNA synthetase (leuS), peroxide stress regulator (per), and are involved with the purine biosynthesis (purH and purN [16-18] (https://www.uniprot.org/uniprot/P67514, www.patri cbrc.org). The genes distribution according to the clinical outcome status of cows from which the strains were isolated is presented on Table 1.

Some genes had low prevalence (less than three cows infected by the isolates positives for each gene). Among the genes statistically associated with reoccurrence of $\mathrm{CM}$ were $\operatorname{atm\mathrm {B}}, c c p \mathrm{~A}, c l p \mathrm{P}, c p s \mathrm{Y}, \operatorname{lux\mathrm {S}}, \operatorname{sod\mathrm {A}}, \mathrm{SP} \_0095$, SP_0320, SP_0829, SP_0916, SP_2086, Spy_1633, and vicK. The genes SP_1970 and SP_0916 were associated with death/culling of the cow during lactation. For clinical cure, cpsY, lepA, sodA, SP_0095, SP_0829, SP_0916, SP_1970 were associated with no cure. No virulence genes were associated with the bacteriological cure. Only the gene SP_0916 was associated with more than two outcomes (risk of reoccurrence, clinical cure and death/ mortality). The genes $\operatorname{sodA}$, cpsY, SP_0829, SP_0095 were associated with reoccurrence and clinical cure. Finally, the gene SP_1970 was associated with mortality and clinical cure (Fig. 3). The Venn diagram (Fig. 3) for virulence, resistance genes and drug target genes was done with the genes that showed statistical significance $(P<0.05)$ for any clinical outcome.

S. uberis has several virulence genes that have an important role in its pathogenicity. Among them we can highlight the hyaluronic acid capsule genes (has A, has $\mathrm{B}$, and $h a s C)$, the plasminogen activator A gene (pauA), and the $S$. uberis adhesion molecule gene (sua) $[16,19,20]$. In our study, all strains presented has $C$, and just one hasA.

The putative membrane-bound protein transports essential amino acids across the cytoplasmic membrane and it is a virulence factor that promote growth of bacteria in milk [21]. The vru cluster co-ordinate the expression of many putative virulence-associated genes during growth of S. uberis in milk [22]. These genes and other genes (pauA, opp, mtuA), singly or as a group, have not been shown to be specifically associated with mastitis; however, there is evidence that certain host-adapted strains of $S$. uberis have enhanced ability to cause clinical mastitis [23-25].

Strains of $S$. uberis isolated from cases of bovine mastitis display variable amounts of hyaluronic acid capsule. Capsule production is dependent of the has operon, which consists of the has $\mathrm{AB}$ gene cluster and 


\begin{tabular}{|c|c|c|c|c|}
\hline & & & P.value & 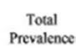 \\
\hline Mannose-6-phosphate isomerase (EC 5030108) & - & $\longrightarrow$ — & 0.03 & 0.685535 \\
\hline tRNA-Asn-GTT & - & $\rightleftarrows$ • & 0.02 & 0.540881 \\
\hline Cysteine desulfurase (EC 2080107), associated with tRNA 4-thiouridine synthase & - & $\longrightarrow$ — & 0.04 & 0.63522 \\
\hline ABC transporter ATP-binding protein & - & $\longrightarrow$ — & 0.03 & 0.559748 \\
\hline Multi antimicrobial extrusion protein ( $\mathrm{Na}(+)$ /drug antiporter), MATE family of MDR efflux pumps & - & $\longrightarrow$ & 0.07 & 0.622642 \\
\hline Putative transmembrane protein & - & $\longrightarrow$ — & 0.04 & 0.440252 \\
\hline 3-oxoacyl-[acyl-carrier-protein] synthase, KASIII (EC 203010180) & - & $\longrightarrow$ — * & 0.05 & 0.45283 \\
\hline Hydrolase, alpha/beta fold family & - & $\longrightarrow$ & 0.16 & 0.698113 \\
\hline DUF402 family nucleoside diphosphatase & - & $\longrightarrow$ & 0.08 & 0.373358 \\
\hline Lipopolysaccharide biosynthesis protein & 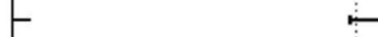 & $\longrightarrow$ & 0.09 & 0.238994 \\
\hline Bis-ABC ATPase YbiT & 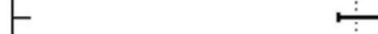 & $\longrightarrow$ & 0.17 & 0.666667 \\
\hline Uncharacterized membrane protein YfhO-like & 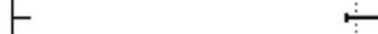 & $\longrightarrow$ & 0.11 & 0.484277 \\
\hline GtrA family protein & $\stackrel{\vdots}{\vdots}$ & $\longrightarrow$ & 0.17 & 0.345912 \\
\hline Sensory transduction protein kinase (EC 207030-) & 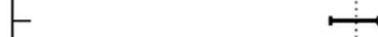 & — & 0.32 & 0.597484 \\
\hline LSU ribosomal maturation GTPase RbgA (B0 subtilis YlqF) & 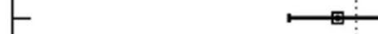 & & 0.46 & 0.446541 \\
\hline PTS system, sucrose-specific IIB component, IIC component, IIA component (EC 207010211) & 0 & & 0.22 & 0.320755 \\
\hline Glutamine--fructose-6-phosphate aminotransferase [isomerizing] (EC 20601016) & — & & 0.12 & 0.383648 \\
\hline Competence-specific sigma factor $\operatorname{ComX}$ & ط * & & 0.04 & 0.698113 \\
\hline 23S rRNA (guanosine(2251)-2'-O)-methyltransferase (EC 201010185) & $\longmapsto$ & & 0.08 & 0.433962 \\
\hline 2-dehydro-3-deoxy-D-gluconate 5-dehydrogenase@ 2-deoxy-D-gluconate 3-dehydrogenase & $\longmapsto$ * & & 0.04 & 0.584906 \\
\hline Phospho-N-acetylmuramoyl-pentapeptide-transferase (EC 20708013) & $\longmapsto$ * * & & 0.04 & 0.553459 \\
\hline FIG086557: Conjugation related protein & $\rightarrow$ & & 0.09 & 0.295597 \\
\hline 3-oxoacyl-[acyl-carrier-protein] synthase, KASII (EC 203010179) & $\longmapsto$ • & & 0.04 & 0,433962 \\
\hline ATP-dependent DNA helicase RecG (EC 30604012) & ط—* & & 0.02 & 0.484277 \\
\hline PTS system, fructose-specific IIA component, IIB component, IIC component (EC 207010202) & 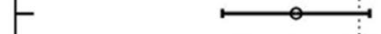 & & 0.09 & 0.257862 \\
\hline 3-oxoacyl-[acyl-carrier protein] reductase (EC 101010100), FadG & $\longmapsto$ — & & 0.02 & 0.490566 \\
\hline Transcriptional regulator, GntR family & 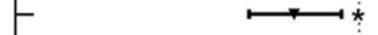 & & 0.006 & 0.811321 \\
\hline Enoyl-[acyl-carrier-protein] reductase [FMN, NADH] (EC 1030109), FabK $\Rightarrow$ refractory to triclosan & $\longrightarrow$ & & 0.005 & 0.528302 \\
\hline phage head-tail adaptor, putative & 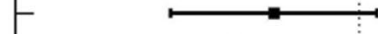 & & 0.11 & 0.176101 \\
\hline Ribosyl nicotinamide transporter, PnuC-like & - & & 0.08 & 0.157233 \\
\hline & 01 & 10. $>$ & & \\
\hline & decreased mortality & mortality & & \\
\hline
\end{tabular}

has $\mathrm{C}$ gene [16]. The has operon comprises the has $\mathrm{A}$ (encoding the hyaluronan synthase), has B (encoding the UDP-glucose dehydrogenase), and has $\mathrm{C}$, which encodes the UDP-glucose pyrophosphorylase [26]. The has A gene product is essential for capsule production in S. uberis [16]. Some studies have reported that because of the capsule absence, $S$. uberis is able to support the bactericidal effect of bovine neutrophils and induce mastitis in dairy cows [27].

The genes $p u r \mathrm{~N}$ e $p u r \mathrm{H}$, which were identified in $100 \%$ of our isolates, are reported to be involved with the purine biosynthesis. These genes are involved with the de novo purine biosynthetic pathway responsible for the synthesis of inosine monophosphate. Studies showed that mutations in purine biosynthetic genes attenuate virulence in Brucella abortus, and it was demonstrated the importance of these genes for growth of several pathogens, as Salmonella enterica and Bacillus anthracis, in human serum $[17,18]$.

\section{Resistance factors}

The emergence of drug resistance by bacteria has been associated with the overuse of antibiotics [28]. At the same time, mastitis is recognized as the main cause for antimicrobial use in dairy farms [2, 29, 30]. Although S. uberis is one of the most important cause of bovine mastitis in dairy herds [31, 32], the resistome of isolates from cows with mastitis demands further understanding. Herein, thirty-four resistance genes were identified in at least one strain. All strains presented $r \operatorname{lm} \mathrm{A}$ (II), rpoB, $r p o C$ genes, although no mutation was observed. For acquired resistance genes, we observed that 152 (95.6\%) strains had a resistance gene to lincosamine $(\ln u \mathrm{D}), 48$ $(30.2 \%)$ to tetracycline $(\operatorname{tet} \mathrm{M}), 4(2.51 \%)$ to tobramicine (ant6), 1 to lincosamide $(l s a(\mathrm{E}))$.

The distribution of the genes according to the clinical outcome following the CM diagnosis is presented in Table 2. The gene pgs $\mathrm{A}$, which is reported to be associated with daptomycin resistance when have a mutation 


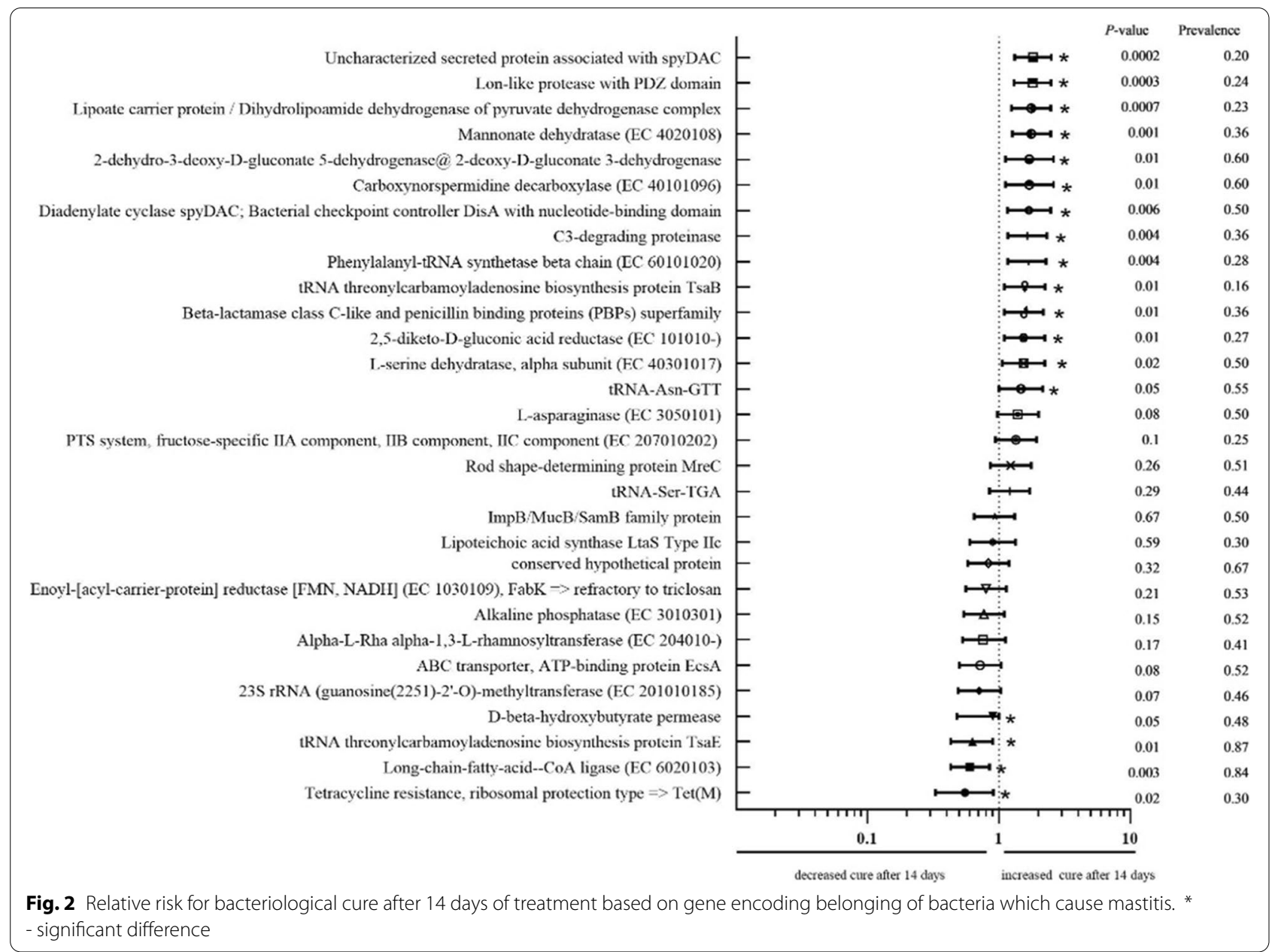

[33], significantly decreased the risk of death/culling. A study identified that the substitution in two enzymes involved in the cardiolipin biosynthesis pathway, i.e., CdsA (phosphatidate cytidylyltransferase) and PgsA (CDP-diacylglycerol-glycerol-3-phosphate-3-phosphatidyltransferase), were associated with no production of phosphatidylglycerol and cardiolipin from cell membranes [34]. Furthermore, the risk of mastitis reoccurrence significantly increased with the presence of several genes $(d d l, f o l \mathrm{P}, g d p \mathrm{D}, g l d \mathrm{~B}, g y r \mathrm{~A}, g y r \mathrm{~B}$, lia $\mathrm{F}$, lia $\mathrm{R}$, liaS, $\ln u \mathrm{D}$, murA).

Previous studies have reported the resistance of $S$. uberis and they demonstrated that it is higher than $S$. dysgalactiae, which is another important Streptococcus causing mastitis in dairy cows [14, 35]. Despite the importance of bacterial resistance, few studies assessed the association between antimicrobial resistance genes of mastitis-causing streptococci and clinical outcomes after intramammary infections [15].

A total of $95.6 \%$ of isolates enrolled in our study presented the gene $\ln u \mathrm{D}$, although it was not associated with clinical outcomes of cows affected with clinical mastitis. The gene $\ln u \mathrm{D}$ was reported to be associated with resistance to lincomycin [36]. The mechanism of action of lincosamides, including lincomycin, is to prevent protein synthesis by inhibiting the peptidyltransferase to several nucleotides of $23 \mathrm{~S}$ rRNA in the $50 \mathrm{~S}$ subunit of the bacterial ribosome. Along with the gene $\ln u \mathrm{D}$, other genes were reported to confer to streptococci resistance to lincosamides, such as $\ln u \mathrm{~B}$ and $\operatorname{lin} \mathrm{B}[15,37,38]$. In our study only two strains presented the InuB and none had the $\operatorname{lin} B$ gene.

The gene $l s a(\mathrm{E})$, identified in one of our strains, was also reported to confer resistance to lincosamide besides streptogramin A and pleuromutilin antibiotics [39]. This gene was also identified in Enterococcus faecalis and Staphylococcus aureus strains, and were reported be located on a multiresistance gene cluster, suggesting that the intra- and inter-genus dissemination and exchange of resistance genes could occur via the plasmids [40]. Therefore, monitoring this gene in gram-positive pathogens causing mastitis, such as $S$. uberis can be relevant to 


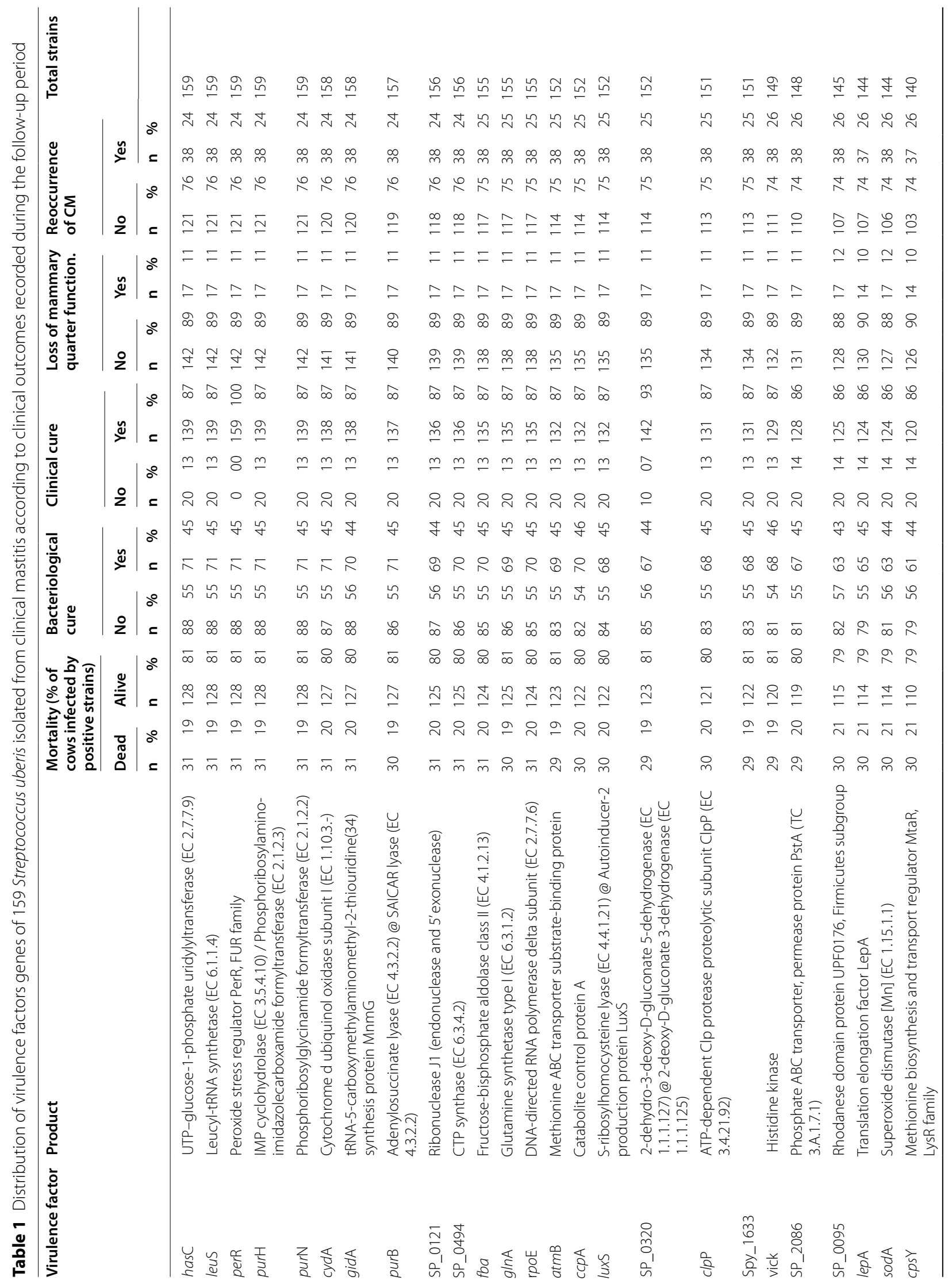




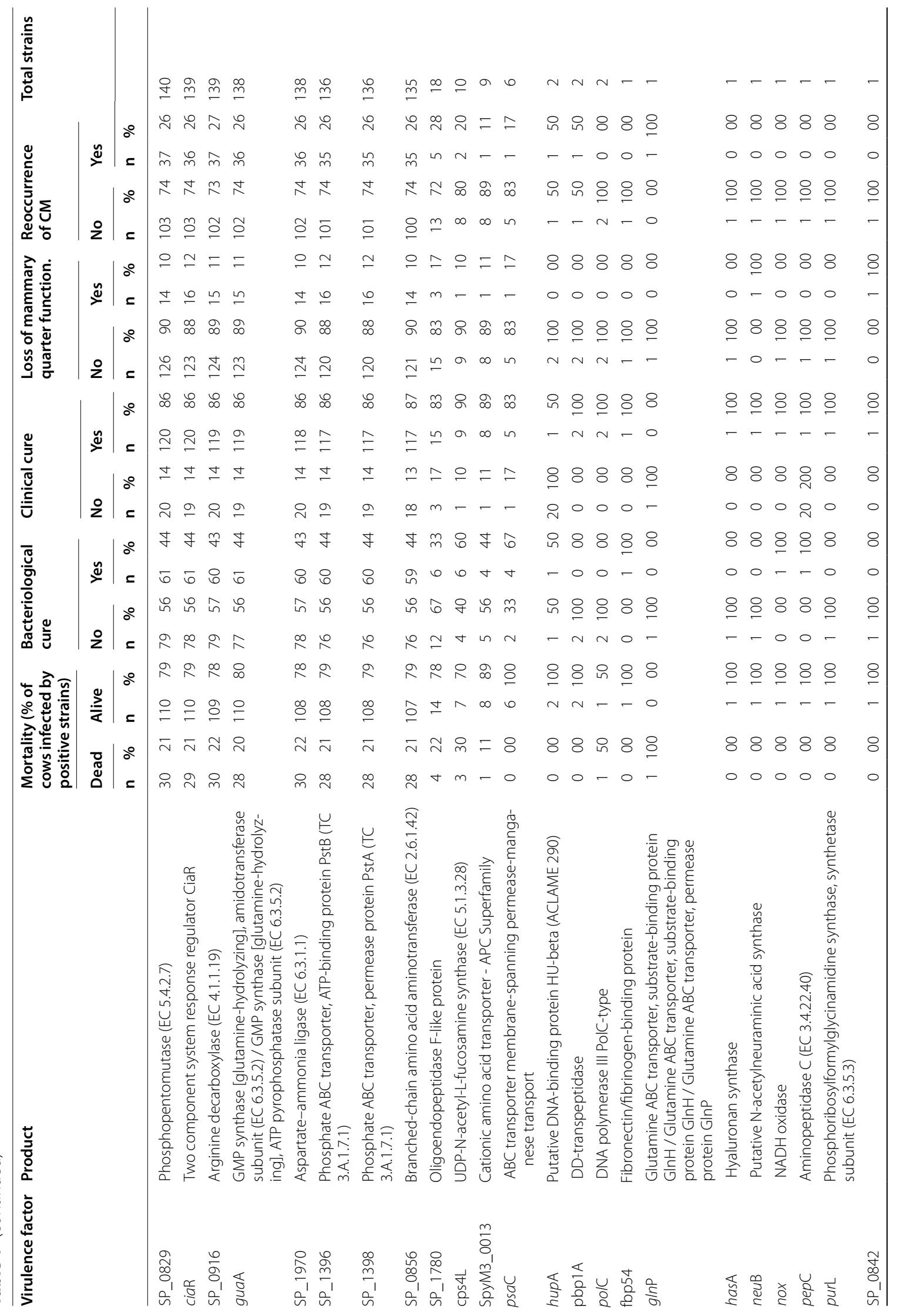




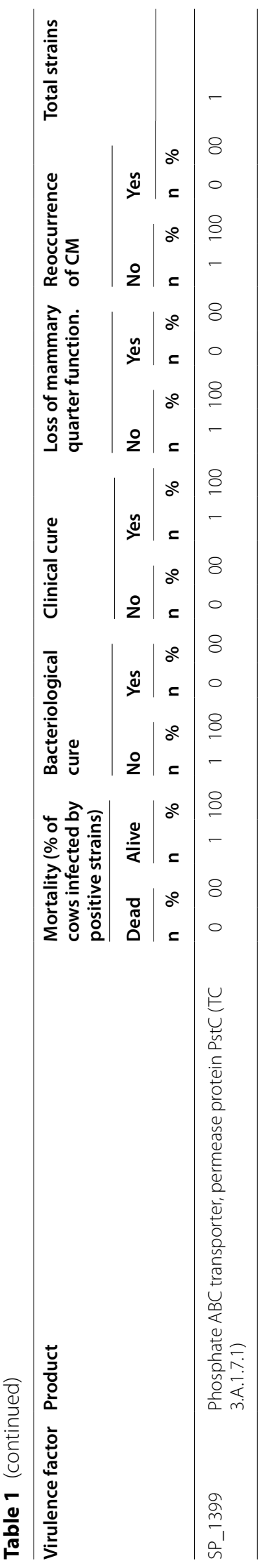




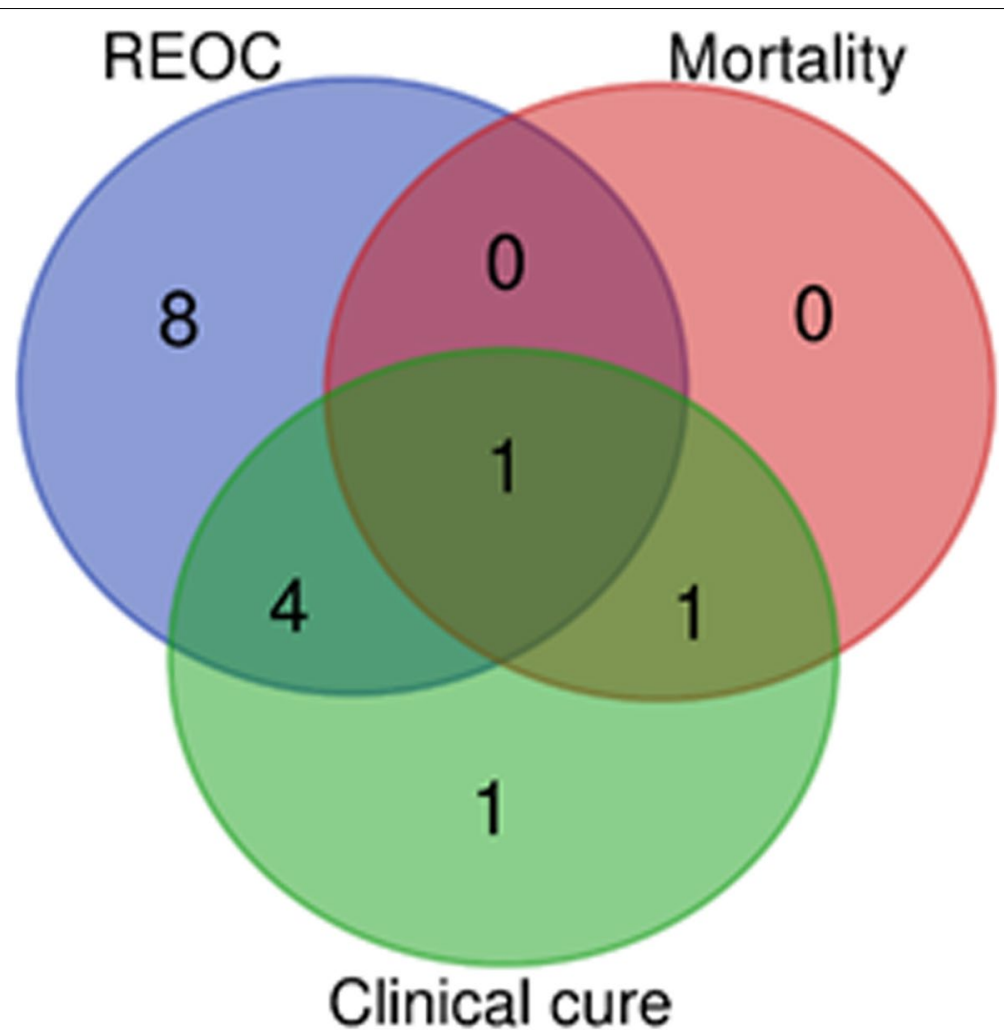

Fig. 3 Venn diagram presenting the frequency of virulence genes of Streptococcus uberis isolated from clinical mastitis that were associated with the clinical outcomes recorded during the follow-up period. REOC: reoccurrence of CM

prevent resistance to antimicrobials used to treat mastitis [40].

Herein, the gene tet $\mathrm{M}$, which is associated with resistance to tetracycline, decreased the risk of bacteriological cure after 14 days of CM diagnosis. In total, $30.2 \%$ of all enrolled strains had the tet $\mathrm{M}$ gene. Recent studies have reported low in vitro susceptibility of $S$. uberis to tetracycline $[13,41]$, which may be attributed to the excessive use of these antimicrobials by the systemic route for treatment of infections in dairy cows and as growth promoter in other species. However, we were not able to indicate any plausible explanation why cows with $\mathrm{CM}$ caused by $S$. uberis having the tetM gene had lower risk of bacteriological cure, especially because tetracycline was not used for CM treatment on the selected herd. Furthermore, this antimicrobial is not labeled for treatment of bovine mastitis in US. Tetracycline is an antimicrobial frequently used systemically to treat respiratory and hoof infection in cattle [30], which may explain the presence of resistant S. uberis strains among our bacteria collection.

Four strains presented the gene ant 6 which confers resistance to a tobramycin, an aminoglycoside with a broad antibacterial spectrum in vitro, and pharmacokinetic properties similar to gentamicin [42]. The resistance to aminoglycosides has clinical importance, since combination of penicillin $\mathrm{G}$ with an aminoglycoside has been recommended for severely ill patients [43]. In addition, penicillin-based products are among the antimicrobials approved to be used for treatment of mastitis in US [44].

The use of antibiotics in food-producing animals can promote the bacterial resistance and allow the presence of antibiotic residues in derived products from animals consumed by human [45].

\section{MLST}

Multi Locus Sequence Type (MLST) is a technique used to analyze constitutive genes based on single nucleotide polymorphism (SNP) (https://pubmlst.org/bigsdb?db= pubmlst_suberis_seqdef).

High genetic diversity was observed in our collection of isolates, which was reflected by a large number of sequence types (STs). In total, eleven distinct STs were observed in our study. Twenty-three strains were classified ST797, which is the only known sequence type among our isolates. Of those, 8 (34.8\%) had bacteriological cure, and 6 (26.1\%) died or were culled during the follow up period. The remained 136 strains did not match with any ST and received a new number. 


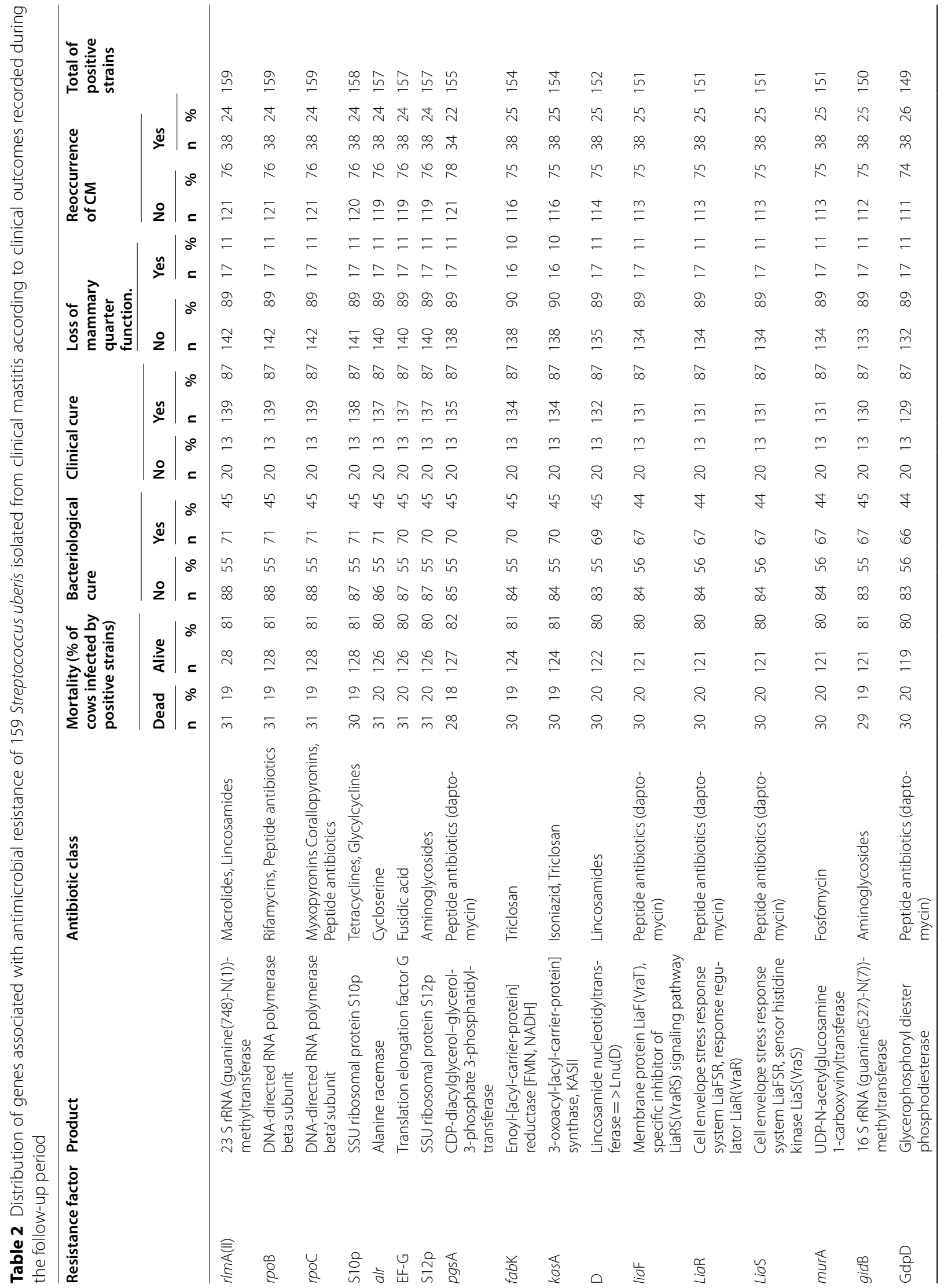




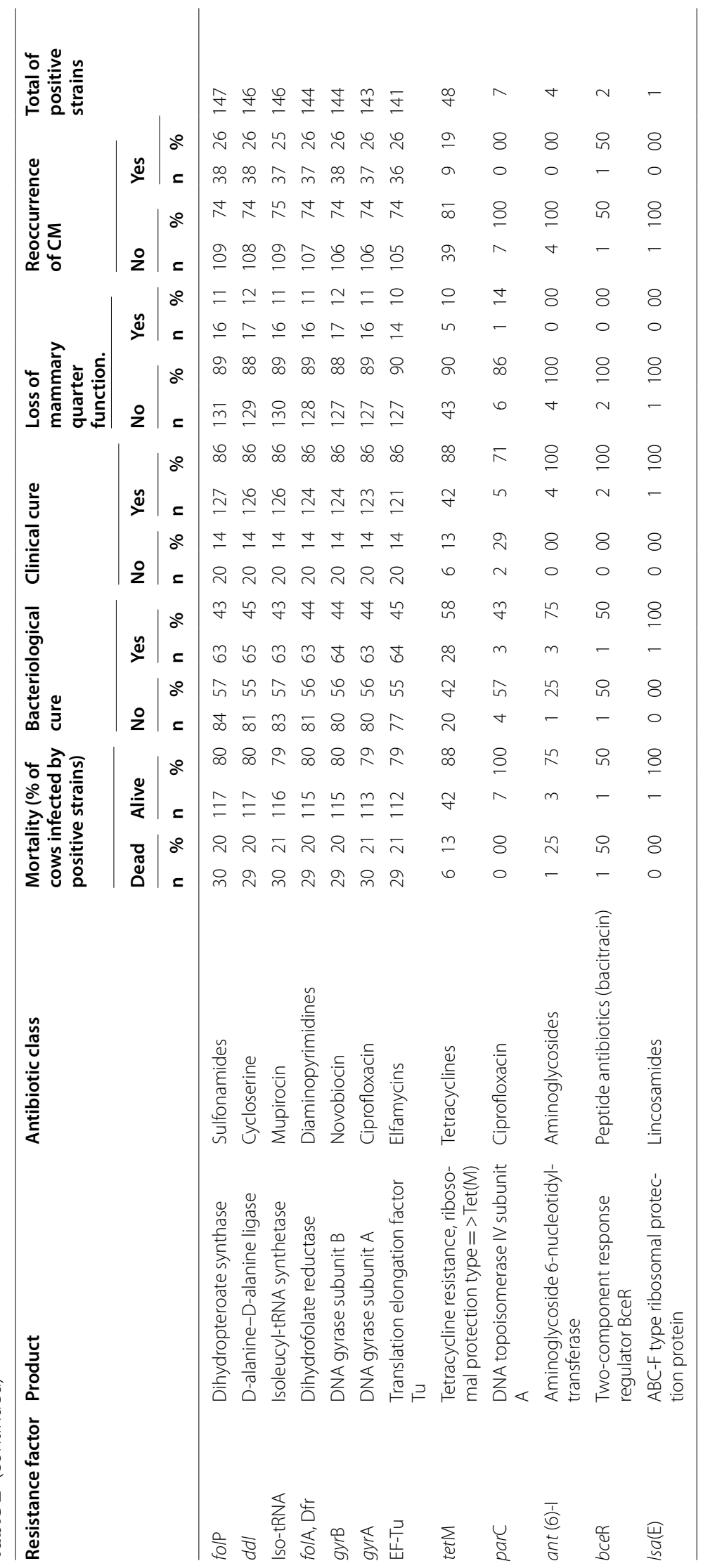


In addition to the ST797, the most identified MLSTtypes were ST1215 $(n=58)$ and ST1219 $(n=35)$. ST1215 was isolated from 58 cows, of which $30(54.5 \%)$ had bacteriological cure, $8(14.5 \%)$ died or were culled, and $5(8.6 \%)$ lost the functionality of the affected mammary quarter. Of the 35 isolates identified as ST1219, $13(37.1 \%)$ had bacteriological cure, 6 (17.1\%) died or were culled. Other types identified from our bacteria collection were ST1213 ( $n=15$ isolates), ST1216 $(n=8)$, ST1221 $(n=7)$, ST1214 $(n=2)$, ST1217 $(n=2)$, ST1218 $(n=2)$, ST1220 $(n=2)$ and ST1212 $(n=1)$. It was not possible to identify the MLST of four strains.

The STs found did not belong to any clonal complex (https://pubmlst.org/bigsdb? db=pubmlst_suberis_ seqdef). The most commons MLST types found were ST1215, ST1219, and ST797. Among them, the cows infected by ST797 showed higher prevalence of death, whereas cows isolated with the ST1215 had higher prevalence of bacteriological cure.

The route of transmission of $S$. uberis has been discussed. Various aspects are related with the routes of transmission, being important to consider the interaction host-pathogen and infection pressure [10]. Although $S$. uberis is one of the main pathogens causing mastitis, its epidemiology is not totally understood. The understanding of epidemiological aspect associated with mastitiscausing S. uberis can help in the development of focused strategies to control this pathogen in dairy farms.

In our study, we observed 10 new STs and one already known. Davies et al., 2016 reported 195 different STs of S. uberis in 52 herds. Only in 10 herds, eleven or more sequence types per herd were observed, showing that in general few STs are related with mastitis within a herd. $71 \%$ of cows were infected by the three more prevalent STs (ST1215, ST1219 and ST797), suggesting that specific strains are more likely to cause mastitis than others are. Although the transmission of S. uberis occur mainly by the environmental route, the transmission from cow to cow can be facilitated in herds with inadequate practices for prevention of contagious pathogens of mastitis (e.g., poor milking routine) [37].

\section{Phylogenetic analysis}

The pangenome of 6,547 unique protein-coding sequences was performed using $159 \mathrm{~S}$. uberis strains enrolled in the study. A total of 29,518 SNPs extracted from the 1,421 core genes was used to infer the ML phylogeny. The results revealed a deep branching and scattered population structure that was broadly classified into distinct phylogenetic lineages, indicating a high genomic diversity of S. uberis isolates studied. The prevalence of ST1215 contributed to the emergence of the unique dominant phylogroup. This lineage included strains isolated from all four mammary quarters, mainly associated with mild clinical score of CM, and with cows with more than 2 lactations. However, two cows infected by the isolates within this phylogroup have died or were culled after clinical mastitis caused by S. uberis. According to clinical outcomes, the isolates were unclustered and intermingled among strains associating with various clinical responses (Fig. 4).

The phylogenetic tree showed that the strains are diverse and, even when they are considered similar, it could yield similar (e.g., strains $186 \mathrm{M}$ and $198 \mathrm{M}$ ) or different clinical outcomes (e.g., strains $95 \mathrm{M}$ and $66 \mathrm{M}$ ).

\section{Conclusions}

In the present study, 159 S. uberis isolates were obtained from cows with clinical mastitis and their genomes were successfully investigated. Virulence and resistance genes were widely identified among isolates and associated with clinical outcomes. Interestingly, from eleven STs identified only one was previously reported, the other ten new STs were documented through this work. Although the strains were isolated from a single herd, they were highly diverse, which confirms S. uberis as an environmental pathogen. Our results can be used as reference for understanding the epidemiology of S. uberis causing mastitis, and also, in future studies targeting the development of new strategies for control and prevention of mastitis caused by this pathogen in dairy herds.

\section{Materials and methods Origin of isolates}

S. uberis were isolated from cases of clinical mastitis identified in a large commercial dairy farm located near Ithaca, New York. The farm milked approximately 4,100 Holstein cows 3 times daily in a 100-stall rotary milking parlor. The animals were housed in freestall barns, with concrete stalls covered with mattresses and bedded with manure solids. The farm had an average milk production per cow of $40.4 \mathrm{Kg}(42.2 \mathrm{Kg}$ of energy corrected milk) and bulk milk SCC of 135.330 cells $/ \mathrm{mL}$ during the period of bacteria isolation.

Farm personnel recorded the severity scores of mastitis as mild (changes in the milk appearance), moderate (changes in the milk appearance associated with udder edema) or severe (presence of systemic signs such as fever, dehydration, prostration) and the distribution of scores was extracted from the farm management software (Dairy Comp 305; Valley Agricultural Software, Tulare, CA).

Strains were identified in a contemporary clinical trial evaluating the efficacy of four protocols for treatment of clinical mastitis caused by Gram-positive pathogens [46]. Briefly, all clinical mastitis cases identified on the farm 


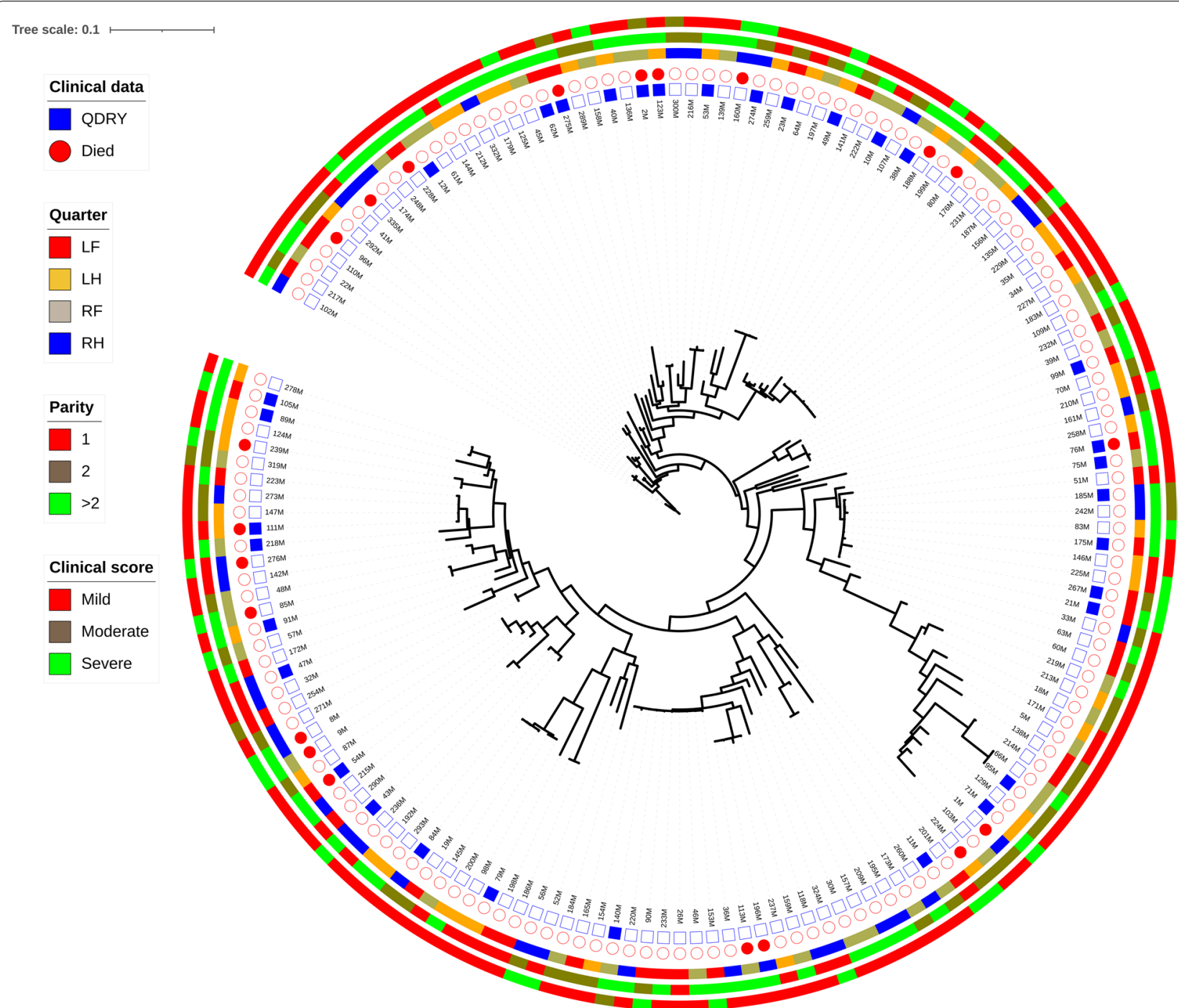

Fig. 4 Phylogeny of core genome SNPs in 159 genomes of Streptococcus uberis isolates from dairy cows with clinical mastitis and according to the clinical data recorded for each case. The RAxML program was used to calculate the phylogenetic tree to construct a maximum likelihood phylogeny

had two milk samples collected using aseptic methods. One milk sample was collected by the herdsmen as part of the farm's selective treatment program of CM, which was submitted for aerobic culture using the guidelines of National Mastitis Council (2017). The second milk sample was collected by the researchers and cultured only if the first sample had identification of S. uberis. In this step, analysis of total Gram-positive bacteria count was performed using the technique of Agar droplets [47] using a selective and differential culture medium (Accutreat ${ }^{\circledR}$, FERA Diagnostics and Biologicals,, Ithaca, NY). Results of bacterial counts are published elsewhere [46]. A single colony was selected from the aforementioned culture plate and streaked onto a CHROMagar Streptococcus base (CHROMagar, France) plate followed by incubation overnight at $37^{\circ} \mathrm{C}$. This procedure was repeated two more times to ensure purity and a single colony was used for further analysis. The strains used in this study were isolated during the aforementioned procedure using the milk samples collected before CM treatment.

The cows were treated with antibiotics within $48 \mathrm{~h}$ after CM identification. As our study was performed contemporarily with another study [48], cows identified with Gram-positive mastitis were randomly allocated to three treatment groups: amoxicillin (label use), 3 infusions with $62.5 \mathrm{mg}$ of amoxicillin (Amoxi-Mast, Merck Animal Health, Millsboro, DE) performed at $12 \pm 2 \mathrm{~h}$ apart; amoxicillin (extra-label), 5 infusions once a day 
with $62.5 \mathrm{mg}$ of amoxicillin (Amoxi-Mast, Merck Animal Health); or ceftiofur (label use), 5 infusions once a day with $125 \mathrm{mg}$ of ceftiofur hydrochloride (Spectramast, Zoetis, Kalamazoo, MI) [46].

Follow up outcomes for cows from which the $S$. uberis strains were originated were registered using the dataset of Tomazi et al. [48]. Briefly, clinical cure was defined as the return of milk to normal appearance according to a clinical examination performed $14 \pm 3$ days after $\mathrm{CM}$ diagnosis. Bacteriological cure was defined as the absence of bacterial growth in milk cultures performed at $14 \pm 3$ days after CM diagnosis. A recurrent case of $\mathrm{CM}$ was defined when a new case occurred in the same quarter from 15 to $90 \mathrm{~d}$ after identification of $\mathrm{CM}$ and the milk culture yielded the same bacterial species isolated at diagnosis. A quarter loss was defined as the loss of mammary quarter physiological function due to the damage caused by the mastitis case. And the culling or death of cows was based on the farm records up to 90 days of $\mathrm{CM}$ diagnosis [46].

\section{Bacterial identification}

The DNA was extracted from each bacterial isolate using DNAasy Power food Microbial Kit (Qiagen, Valencia, CA, USA) following the manufacturer's instructions. NanoDrop ND-1000 spectrophotometer (NanoDrop Technologies, Rockland, DE) was used for DNA quantification. Then, a PCR for the $16 \mathrm{~S}$ ribosomal DNA gene amplification was performed using a mix constituted of: 10 pmol of each fD1 forward and rP2 reverse primers [48], Econo-Taq Plus Green $1 \times$ Master Mix (Lucigen, Middleton, WI), 280 to $350 \mathrm{ng}$ of template DNA, and ultrapure distilled water (added to complete the volume to $100 \mu \mathrm{L}$ ). The parameters used for amplification were $94{ }^{\circ} \mathrm{C}$ for $5 \mathrm{~min}, 57^{\circ} \mathrm{C}$ for $2 \mathrm{~min}$, and $72{ }^{\circ} \mathrm{C}$ for $2 \mathrm{~min}$ followed by 29 cycles of $94{ }^{\circ} \mathrm{C}$ for $2 \mathrm{~min}, 57^{\circ} \mathrm{C}$ for $30 \mathrm{~s}$, and $72{ }^{\circ} \mathrm{C}$ for $2 \mathrm{~min}$, with a final extension of $72{ }^{\circ} \mathrm{C}$ for $10 \mathrm{~min}$ [49]. The presence of PCR products was confirmed by agarose gel electrophoresis $(1.2 \% \mathrm{wt} / \mathrm{vol})$ with $0.5 \mu \mathrm{g} /$ $\mathrm{mL}$ ethidium bromide. The PCR products were purified using Gel/PCR Fragments Extraction Kit (IBI Scientific, Peosta, IA) following the manufacturer's recommendations. The purified DNA samples were submitted to the Cornell University Institute of Biotechnology for Sanger sequencing using 8 pmol of primer fD1 and 300 ng of PCR products. For identification of species, we compared our FASTA sequences with the sequences stored in GenBank, using the BLAST algorithm (http://blast.ncbi.nlm. nih.gov/Blast.cgi).

\section{Whole-genome sequencing}

Samples were diluted by adding UltraPure Water (Invitrogen, Waltham, MA) until a concentration of $0.2 \mathrm{ng} /$ $\mu \mathrm{l}$, measured using a Qubit fluorometer (Thermo Fisher Scientific, Waltham, MA). After normalization, the samples were used as an input to the Nextera XT DNA Sample Prep Kit (Illumina Inc. San Diego, CA). The library preparation was done according to the manufacturer's protocol (Nextera ${ }^{\circledR}$ DNA Library Prep Reference Guide). Tagmentation of samples was done using $1 \mathrm{ng}$ of template, then PCR amplification was done using a unique combination of barcode primers (provided by manufacture). The purification of libraries was performed using Mag-Bind Totalpure NGS (Omega BioTek - Norcross, GA) bead purification and then normalized through Library Normalization beads/additives. For preparation of cluster generation and sequencing, equal volumes of normalized libraries were combined, diluted in hybridization buffer and heat denatured. Finally, we performed pair-end sequencing using a MiSeq Reagent Kit v3 (600 cycles) in the Illumina MiSeq platform.

\section{Genome sequence analyzing}

The quality of the original reads was evaluated using FASTQC. The potential contamination of sequences was checked by Kraken2 (Taxonomic sequence classification system) [50].

The sequencing reads were submitted to the comprehensive genome analysis service using Pathosystems Resource Integration Center (PATRIC 3.2.96) [51]. The reads were assembled using SPAdes [52] and the genomes were annotated using the Rast tool kit available in the PATRIC system, as part of the all-bacteria Bioinformatics Resource Center available online [53]. In silico multilocus sequence typing (MLST) was performed by MLST 1.8 (https://cge.cbs.dtu.dk/services/MLST/). Acquired antibiotic resistance genes (ARGs) were identified using ABRicate version 0.5 (https:/github.com/ tseemann/abricate) by aligning genome sequences to the ResFinder database [54]. Virulence genes were identified using VFDB database [55]. Plasmid replicon types were detected using PlasmidFinder v1.3. [56]. The IS elements were confirmed by searching in ISFinder (https://wwwisfinder.biotoul.fr).

\section{Phylogenetic analysis}

For each de novo assembly, coding sequences were predicted using Prodigal v. 2.6 [57] and annotated using the rapid prokaryotic genome annotation tool, Prokka [58]. The core genes were identified and used to build the core genome using Roary [59] with the -e -mafft setting to create a concatenated alignment of core genomic CDS. SNP-sites (https://github.com/sanger-pathogens/ snp-sites) was used to extract the core genomic SNPs [60]. To construct a maximum likelihood phylogeny of the sequencing isolates, RAxML was used with the 
generalized time-reversible model and a GTRGAMMA distribution to model site-specific rate variation [61]. Support for the ML phylogeny was assessed by 100 bootstrap pseudo-analyses of the alignment data. We used iTOL [62] and FigTree (www.tree.bio.ed.ac.uk/software/ figtree/) to visualize and edit the phylogenetic tree.

\section{Statistical analysis}

Descriptive analysis of gene frequency and distribution of genes according to treatment outcomes was performed using JMP PRO 14 (SAS Institute Inc., Cary, NC). Using JMP Pro 14, we selected 30 most important encoding gene through of Predictor Screening and we used 100,000 trees to make the analyzes. We used this to predict the most important genes for variables mortality and bacteriological cure in 14 days. Med Calc was used to calculate risk relative of each variable and prism (GraphPad) was used for plot data.

The Venn diagram was performed using the website: http://bioinformatics.psb.ugent.be/webtools/Venn/ .

\section{Acknowledgements}

This work was supported by São Paulo Research Foundation - FAPESP.

\section{Authors' contributions}

N.C.C.S and M.X.R. performed the analyzes bacteria isolation and DNA sequencing and wrote the manuscript, T.T. collected the data samples and farm data, and revised the manuscript; Y.Y. made the part of bioinformatic analysis, and revised and corrected the manuscript; R.C.B. contributed to conception and design, conceived the study and performed data analysis and interpretation of data. The author(s) read and approved the final manuscript.

\section{Funding}

grant \#2018/24191-3, São Paulo Research Foundation (FAPESP).

\section{Availability of data and materials}

The datasets generated and/or analysed analyzed during the current study are available in database from at www.patricbrc.org and directly from the corresponding author on reasonable request.

\section{Declarations}

\section{Ethics approval and consent to participate}

This study was carried out in strict accordance with the recommendations of The Animal Welfare Act of 1985 (P.L. 99-198). The research protocol was reviewed and approved by the Institutional Animal Care and Use Committee of Cornell University (protocol number 2018-0097).

\section{Consent for publication}

Not applicable for that section.

\section{Competing interests}

The authors declare that they have no competing interests.

\section{Author details}

${ }^{1}$ Department of Population Medicine and Diagnostic Sciences, College of Veterinary Medicine, Cornell University, Ithaca, New York, USA. ${ }^{2}$ Department of Food Science and Nutrition, Faculty of Food Engineering (FEA), University of Campinas (UNICAMP), Rua Monteiro Lobato, 80, SP 13083-862 Campinas, Brazil. ${ }^{3}$ Department of Microbiology, Zhongshan School of Medicine, Sun Yatsen University, 510080 Guangzhou, China. ${ }^{4}$ School of Pharmaceutical Sciences (Shenzhen), Sun Yat-sen University, 510006 Guangzhou, China.
Received: 26 April 2021 Accepted: 17 September 2021

Published online: 07 October 2021

\section{References}

1. Hillerton JE, Berry EA. Treating mastitis in the cow-a tradition or an archaism. J Appl Microbiol. 2005;98(6):1250-5.

2. Gomes F, Henriques M. Control of Bovine Mastitis: Old and Recent Therapeutic Approaches. Curr Microbiol. 2016;72(4):377-82.

3. Lundberg $\AA$, Nyman A, Unnerstad HE, Waller KP. Prevalence of bacterial genotypes and outcome of bovine clinical mastitis due to Streptococcus dysgalactiae and Streptococcus uberis. Acta Vet Scand. 2014;56(1):80.

4. Rossi RS, Amarante AF, Correia LBN, Guerra ST, Nobrega DB, Latosinski GS, et al. Diagnostic accuracy of Somaticell, California Mastitis Test, and microbiological examination of composite milk to detect Streptococcus agalactiae intramammary infections. J Dairy Sci. 2018;101(11):10220-9.

5. Ruegg PL. New perspectives in udder health management. Vet Clin North Am Food Anim Pract. 2012;28(2):149-63.

6. de Haas Y, Barkema HW, Veerkamp RF. The effect of pathogen-specific clinical mastitis on the lactation curve for somatic cell count. J Dairy Sci. 2002;85(5):1314-23.

7. Pearson LJ, Williamson JH, Turner SA, Lacy-Hulbert SJ, Hillerton JE. Peripartum infection with Streptococcus uberis but not coagulase-negative staphylococci reduced milk production in primiparous cows. J Dairy Sci. 2013;96(1):158-64.

8. Kester HJ, Sorter DE, Hogan JS. Activity and milk compositional changes following experimentally induced Streptococcus uberis bovine mastitis. J Dairy Sci. 2015;98(2):999-1004.

9. Zadoks RN, Gillespie BE, Barkema HW, Sampimon OC, Oliver SP, Schukken YH. Clinical, epidemiological and molecular characteristics of Streptococcus uberis infections in dairy herds. Epidemiol Infect. 2003;130(2):335-49.

10. Davies PL, Leigh JA, Bradley AJ, Archer SC, Emes RD, Green MJ. Molecular epidemiology of streptococcus uberis clinical mastitis in dairy herds: strain heterogeneity and transmission. J Clin Microbiol. 2016;54(1):68-74.

11. Leelahapongsathon K, Schukken YH, Pinyopummintr T, Suriyasathaporn W. Comparison of transmission dynamics between Streptococcus uberis and Streptococcus agalactiae intramammary infections. J Dairy Sci. 2016;99(2):1418-26.

12. Tamilselvam B, Almeida RA, Dunlap JR, Oliver SP. Streptococcus uberis internalizes and persists in bovine mammary epithelial cells. Microb Pathog. 2006:40(6):279-85.

13. Tomazi T, Freu G, Alves BG, de Souza Filho AF, Heinemann MB, Veiga dos Santos M. Genotyping and antimicrobial resistance of Streptococcus uberis isolated from bovine clinical mastitis. PLOS ONE. 2019;14(10):e0223719.

14. Cameron M, Saab M, Heider L, McClure JT, Rodriguez-Lecompte JC, Sanchez J. Antimicrobial susceptibility patterns of environmental streptococci recovered from bovine milk samples in the maritime provinces of Canada. Front Vet Sci. 2016:3:79.

15. Vélez JR, Cameron M, Rodríguez-Lecompte JC, Xia F, Heider LC, Saab M, et al. Whole-genome sequence analysis of antimicrobial resistance genes in streptococcus uberis and streptococcus dysgalactiae isolates from Canadian dairy herds. Front Vet Sci. 2017;4:63.

16. Ward PN, Field TR, Ditcham WG, Maguin E, Leigh JA. Identification and disruption of two discrete loci encoding hyaluronic acid capsule biosynthesis genes hasA, hasB, and hasC in Streptococcus uberis. Infect Immun. 2001;69(1):392-9.

17. Alcantara RB, Read RD, Valderas MW, Brown TD, Roop RM, 2nd. Intact purine biosynthesis pathways are required for wild-type virulence of Brucella abortus 2308 in the BALB/c mouse model. Infect Immun. 2004;72(8):4911-7.

18. Samant S, Lee H, Ghassemi M, Chen J, Cook JL, Mankin AS, et al. Nucleotide biosynthesis is critical for growth of bacteria in human blood. PLoS Pathog. 2008;4(2):e37.

19. Fang W, Almeida RA, Oliver SP. Effects of lactoferrin and milk on adherence of Streptococcus uberis to bovine mammary epithelial cells. Am J Vet Res. 2000;61 (3):275-9.

20. Oliver SP, Almeida RA, Calvinho LF. Virulence factors of Streptococcus uberis isolated from cows with mastitis. Zentralbl Veterinarmed B. 1998;45(8):461-71. 
21. Taylor DL, Ward PN, Rapier CD, Leigh JA, Bowler LD. Identification of a differentially expressed oligopeptide binding protein (OppA2) in Streptococcus uberis by representational difference analysis of cDNA. J Bacteriol. 2003;185(17):5210-9.

22. Egan SA, Ward PN, Watson M, Field TR, Leigh JA. Vru (Sub0144) controls expression of proven and putative virulence determinants and alters the ability of Streptococcus uberis to cause disease in dairy cattle. Microbiology. 2012;158(Pt 6):1581-92.

23. Tomita T, Meehan B, Wongkattiya N, Malmo J, Pullinger G, Leigh J, et al. Identification of Streptococcus uberis multilocus sequence types highly associated with mastitis. Appl Environ Microbiol. 2008;74(1):114-24.

24. Lang P, Lefébure T, Wang W, Zadoks RN, Schukken Y, Stanhope MJ. Gene content differences across strains of Streptococcus uberis identified using oligonucleotide microarray comparative genomic hybridization. Infect Genet Evol. 2009;9(2):179-88.

25. Tassi R, McNeilly TN, Fitzpatrick JL, Fontaine MC, Reddick D, Ramage C, et al. Strain-specific pathogenicity of putative host-adapted and nonadapted strains of Streptococcus uberis in dairy cattle. J Dairy Sci. 2013;96(8):5129-45.

26. Ward PN, Holden MTG, Leigh JA, Lennard N, Bignell A, Barron A, et al. Evidence for niche adaptation in the genome of the bovine pathogen Streptococcus uberis. BMC Genomics. 2009;10(1):54.

27. Field TR, Ward PN, Pedersen LH, Leigh JA. The hyaluronic acid capsule of Streptococcus uberis is not required for the development of infection and clinical mastitis. Infect Immun. 2003;71(1):132-9.

28. Llor C, Bjerrum L. Antimicrobial resistance: risk associated with antibiotic overuse and initiatives to reduce the problem. Ther Adv Drug Saf. 2014;5(6):229-41.

29. Bengtsson B, Unnerstad HE, Ekman T, Artursson K, Nilsson-Öst M, Waller KP. Antimicrobial susceptibility of udder pathogens from cases of acute clinical mastitis in dairy cows. Veterinary Microbiology. 2009;136(1):142-9.

30. Pol M, Ruegg PL. Treatment practices and quantification of antimicrobial drug usage in conventional and organic dairy farms in Wisconsin. J Dairy Sci. 2007;90(1):249-61.

31. Tomazi T, Ferreira GC, Orsi AM, Goncalves JL, Ospina PA, Nydam DV, et al. Association of herd-level risk factors and incidence rate of clinical mastitis in 20 Brazilian dairy herds. Prev Vet Med. 2018;161:9-18.

32. Verbeke J, Piepers S, Supre K, De Vliegher S. Pathogen-specific incidence rate of clinical mastitis in Flemish dairy herds, severity, and association with herd hygiene. J Dairy Sci. 2014;97(11):6926-34.

33. Hines KM, Waalkes A, Penewit K, Holmes EA, Salipante SJ, Werth BJ, et al. Characterization of the mechanisms of daptomycin resistance among gram-positive bacterial pathogens by multidimensional Lipidomics. mSphere. 2017;2(6):e00492-17.

34. Tran TT, Mishra NN, Seepersaud R, Diaz L, Rios R, Dinh AQ, et al. Mutations in cdsA and pgsA Correlate with Daptomycin Resistance in Streptococcus mitis and S. oralis. Antimicrob Agents Chemother. 2019;63(2):e01531-18.

35. Minst K, Märtlbauer E, Miller T, Meyer C. Short communication: Streptococ cus species isolated from mastitis milk samples in Germany and their resistance to antimicrobial agents. J Dairy Sci. 2012;95(12):6957-62.

36. Petinaki E, Guérin-Faublée V, Pichereau V, Villers C, Achard A, Malbruny B, et al. Lincomycin resistance gene Inu(D) in Streptococcus uberis. Antimicrob Agents Chemother. 2008;52(2):626-30.

37. Rato MG, Bexiga R, Florindo C, Cavaco LM, Vilela CL, Santos-Sanches I. Antimicrobial resistance and molecular epidemiology of streptococci from bovine mastitis. Vet Microbiol. 2013;161(3-4):286-94.

38. Schmitt-Van de Leemput E, Zadoks RN. Genotypic and phenotypic detection of macrolide and lincosamide resistance in Streptococcus uberis. J Dairy Sci. 2007;90(11):5089-96.

39. Berbel D, Càmara J, García E, Tubau F, Guérin F, Giard J-C, et al. A novel genomic island harbouring $I s a(E)$ and $\ln u(B)$ genes and a defective prophage in a Streptococcus pyogenes isolate resistant to lincosamide, streptogramin A and pleuromutilin antibiotics. Int J Antimicrob Agents. 2019;54(5):647-51.

40. Li X-S, Dong W-C, Wang X-M, Hu G-Z, Wang Y-B, Cai B-Y, et al. Presence and genetic environment of pleuromutilin-lincosamide-streptogramin A resistance gene $\mathrm{Isa}(\mathrm{E})$ in enterococci of human and swine origin. J Antimicrob Chemother. 2013;69(5):1424-6.

41. Kaczorek E, Malaczewska J, Wojcik R, Rekawek W, Siwicki AK. Phenotypic and genotypic antimicrobial susceptibility pattern of Streptococcus spp. isolated from cases of clinical mastitis in dairy cattle in Poland. J Dairy Sci. 2017;100(8):6442-53.

42. Brogden RN, Pinder RM, Sawyer PR, Speight TM, Avery GS. Tobramycin: a review of its antibacterial and pharmacokinetic properties and therapeutic use. Drugs. 1976;12(3):166-200.

43. Galimand M, Lambert T, Gerbaud G, Courvalin P. High-level aminoglycoside resistance in the beta-hemolytic group G Streptococcus isolate BM2721. Antimicrob Agents Chemother. 1999;43(12):3008-10.

44. Ruegg PL. What is success? a narrative review of research evaluating outcomes of antibiotics used for treatment of clinical mastitis. Front Vet Sci. 2021;8:639641.

45. Manyi-Loh C, Mamphweli S, Meyer E, Okoh A. Antibiotic use in agriculture and its consequential resistance in environmental sources: potential public health implications. Molecules. 2018;23(4):795.

46. Tomazi T, Sumnicht M, Tomazi ACCH, Silva JCC, Bringhenti L, Duarte LM, et al. Negatively controlled, randomized clinical trial comparing different antimicrobial interventions for treatment of clinical mastitis caused by gram-positive pathogens. J Dairy Sci. 2021;104(3):3364-85.

47. Sharpe AN, Kilsby DC. A rapid, inexpensive bacterial count technique using agar droplets. J Appl Bacteriol. 1971;34(2):435-40.

48. Weisburg WG, Barns SM, Pelletier DA, Lane DJ. 16S ribosomal DNA amplification for phylogenetic study. J Bacteriol. 1991;173(2):697-703.

49. Wood J, Scott KP, Avgustin G, Newbold CJ, Flint HJ. Estimation of the relative abundance of different Bacteroides and Prevotella ribotypes in gut samples by restriction enzyme profiling of PCR-amplified 16S rRNA gene sequences. Appl Environ Microbiol. 1998;64(10):3683-9.

50. Wood DE, Lu J, Langmead B. Improved metagenomic analysis with Kraken 2. Genome Biol. 2019;20(1):257.

51. Wattam AR, Davis JJ, Assaf R, Boisvert S, Brettin T, Bun C, et al. Improvements to PATRIC, the all-bacterial Bioinformatics Database and Analysis Resource Center. Nucleic Acids Res. 2017:45(D1):D535-d42.

52. Bankevich A, Nurk S, Antipov D, Gurevich AA, Dvorkin M, Kulikov AS, et al. SPAdes: a new genome assembly algorithm and its applications to singlecell sequencing. J Comput Biol. 2012;19(5):455-77.

53. Brettin T, Davis JJ, Disz T, Edwards RA, Gerdes S, Olsen GJ, et al. RASTtk: a modular and extensible implementation of the RAST algorithm for building custom annotation pipelines and annotating batches of genomes. Sci Rep. 2015;5:8365.

54. Zankari E, Hasman H, Cosentino S, Vestergaard M, Rasmussen S, Lund O, et al. Identification of acquired antimicrobial resistance genes. J Antimicrob Chemother. 2012;67(11):2640-4.

55. Chen L, Zheng D, Liu B, Yang J, Jin Q. VFDB 2016: hierarchical and refined dataset for big data analysis-10 years on. Nucleic Acids Res. 2016:44(D1):D694-7.

56. Carattoli A, Zankari E, Garcia-Fernandez A, Larsen MV, Lund O, Villa L, et al. In Silico detection and typing of plasmids using PlasmidFinder and Plasmid Multilocus sequence typing. Antimicrob Agents Chemother. 2014;58(7):3895-903.

57. Hyatt D, Chen GL, Locascio PF, Land ML, Larimer FW, Hauser L. Prodigal: prokaryotic gene recognition and translation initiation site identification. BMC Bioinformatics. 2010;11:119.

58. Seemann T. Prokka: rapid prokaryotic genome annotation. Bioinformatics. 2014:30(14):2068-9.

59. Page AJ, Cummins CA, Hunt M, Wong VK, Reuter S, Holden MT, et al. Roary: rapid large-scale prokaryote pan genome analysis. Bioinformatics. 2015;31(22):3691-3.

60. Page AJ, Taylor B, Delaney AJ, Soares J, Seemann T, Keane JA, et al. SNP-sites: rapid efficient extraction of SNPs from multi-FASTA alignments. Microb Genom. 2016;2(4):e000056.

61. Stamatakis A. RAxML-VI-HPC: maximum likelihood-based phylogenetic analyses with thousands of taxa and mixed models. Bioinformatics. 2006;22(21):2688-90.

62. Letunic I, Bork P. Interactive tree of life (iTOL) v3: an online tool for the display and annotation of phylogenetic and other trees. Nucleic Acids Res. 2016;44(W1):W242-5.

\section{Publisher's Note}

Springer Nature remains neutral with regard to jurisdictional claims in published maps and institutional affiliations. 